\section{Stratified medicine in psychiatry: a worrying example or new opportunity in the treatment of anxiety?}

\author{
David R Owen ${ }^{1}$, Rainer Rupprecht ${ }^{2}$ and David J Nutt ${ }^{1}$
}

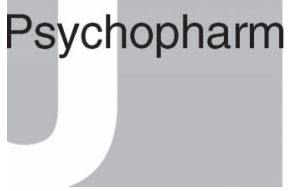

Journal of Psychopharmacology 27(2) 119-122

(c) The Author(s) 2013 Reprints and permission: sagepub.co.uk/journalsPermissions.nav DOI: $10.1177 / 0269881112443746$ jop.sagepub.com

(S)AGE

\begin{abstract}
Stratified medicine is a new term that figures highly in current MRC and NHS strategy. It has developed from the earlier terms individualised or personalised medicine and refers to the use of genetic and/or endophenotypic measures to allow better targeting of treatments. The best exemplar is HER2 positivity in breast cancer to determine the efficacy of Herceptin. Clinical trials of this anti-cancer drug were initially unpromising, but once the HER2 positive subgroup was identified it was found, in this subgroup only, to be highly effective. It is presumed that similar subgroups will be found for many common disorders not just cancers, and that these will lead to much better targeted treatments. Such an advance may be necessary to develop new treatments in certain fields where the development of broad-spectrum/blockbuster treatments appears to have reached the end of the road; a particular example of this is in psychiatry. In this paper we discuss this issue in relation to psychiatry using a new and interesting example of how genotyping might help rescue an apparently failed novel treatment in anxiety disorders.
\end{abstract}

\title{
Keywords
}

Stratified medicine, translocator protein, anxiety, neurosteroids

Anxiety disorders are common and disabling chronic conditions (Nutt et al., 2002). Benzodiazepines are effective drugs (Baldwin et al., 2005) but their side effects (including sedation, development of tolerance and withdrawal) preclude long term use (Baldwin et al., 2005; Nutt et al., 2002). Hence, antidepressants are first-line treatment for many anxiety disorders, but their onset of action is slow, taking weeks to produce an effect (Baldwin et al., 2005; Nutt et al., 2002). Therefore, there remains a need for fast acting anxiolytic agents which lack the unfavourable side effects characteristic of benzodiazepines.

The Translocator Protein (18kDa) (TSPO) has emerged as a potential drug target in the treatment of anxiety disorders (Rupprecht et al., 2010). It is expressed on the outer mitochondrial membrane (OMM) (Woods et al., 1996) of virtually all mammalian cells, and found in highest density in steroidogenic cells (Gavish et al., 1999). Here, it is responsible for an early necessary step in the steroid synthesis pathway: the movement of cholesterol across the cytosolic side of the OMM, to the inner mitochondrial membrane (IMM) (Rone et al., 2009). This brings cholesterol into contact with a side chain cleavage enzyme in the IMM which converts it into pregnenolone, the precursor of all steroids. Pregnenolone subsequently proceeds down a specific synthetic pathway (dependent upon the cell type and hence the enzymes present) to yield the final steroid product. The movement of cholesterol by TSPO is a necessary step in the formation of steroids, and if TSPO is experimentally silenced the rate of steroid synthesis becomes negligible (Papadopoulos et al., 1997).

Steroid synthesis is not restricted to classically recognised steroidogenic tissue such as gonads and the adrenal gland, but in fact occurs within various other cells and tissues including those of the central nervous system (CNS) (Baulieu et al., 2001). Their products, neurosteroids, act at the level of gene expression but are also positive allosteric modulators of neurotransmitter receptors including $\gamma$-aminobutyric acid type $\mathrm{A}\left(\mathrm{GABA}_{\mathrm{A}}\right)$ receptors (Rupprecht et al., 1999). Hence, as might be predicted, neurosteroids cause pronounced anxiolysis in animal models (Brot et al., 1997; Carboni et al., 1996). This, and the observation that their concentrations are reduced during panic attacks in patients with panic disorder (Strohle et al., 2003), suggests that modulating the neurosteroid pathway may have therapeutic potential.

The TSPO is an attractive target for modulating the neurosteroid pathway because movement of cholesterol into the mitochondria is thought to represent the rate limiting step in steroid synthesis (Figure 1) (Papadopoulos, 1993). Indeed, TSPO ligands have been shown to enhance neurosteroidogenesis in the brain and to exert acute anxiolytic activity in rodents (Verleye et al., 2005). The TSPO agonist, XBD173, has been investigated in healthy male volunteers using the cholecystokinin tetrapeptide (CCK4) challenge - a human model of panic-like anxiety (Strohle et al., 2003). Subjects that showed a clear panic response to the neuropeptide CCK 4 were randomised into one of five treatment arms ( $n=14$ in each group) which consisted of seven days' treatment with either placebo, the benzodiazepine alprazolam, or one of

${ }^{1}$ Neuropsychopharmacology Unit, Division of Experimental Medicine, Imperial College London, London, UK

2Department of Psychiatry and Psychotherapy, University Regensburg,

Regensburg, Germany

Corresponding author:

David Owen, Neuropsychopharmacology Unit, Division of Experimental Medicine, Imperial College London, Hammersmith Hospital, Du Cane Road, London, W12 0NN, UK.

Email: d.owen@imperial.ac.uk 


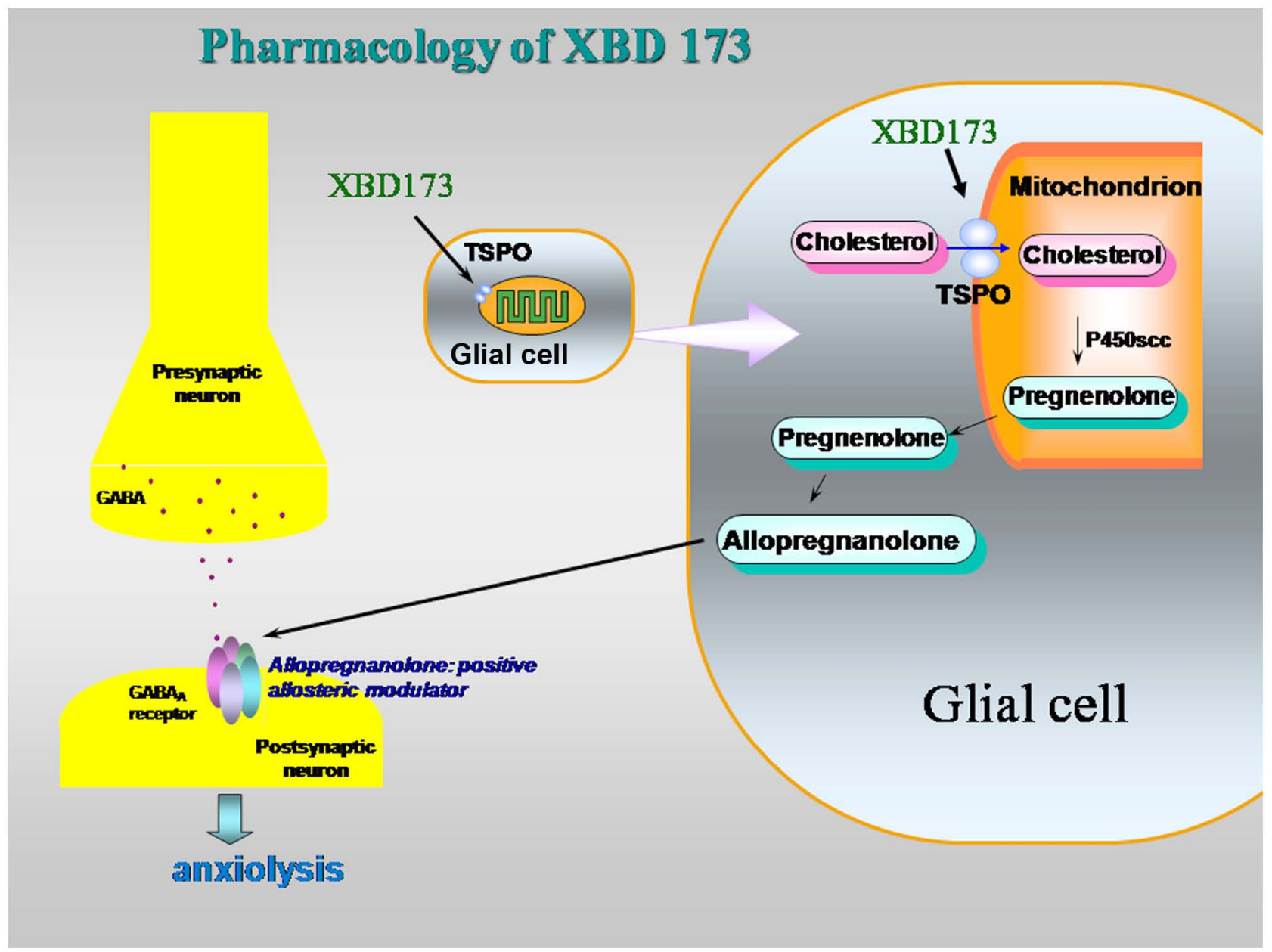

Figure 1. XBD173: mechanism of action.

three doses of XBD173 (10, 30 or $90 \mathrm{mg}$ ). A second CCK4 challenge was performed following the dosing period. At the highest dose, XBD173 attenuated CCK4-induced anxiety compared to placebo $(p<0.036)$ to a degree similar in magnitude to the benzodiazepine (Rupprecht et al., 2009). However, whilst the TSPO agonist matched the benzodiazepine in terms of anxiolysis, the side effects reported were far fewer, and in fact similar to that reported in the placebo group. Whilst $\sim 60 \%$ of subjects treated with the benzodiazepine complained of withdrawal symptoms such as sleep disturbances or restlessness, these side effects were rare in the TSPO agonist groups (Rupprecht et al., 2009).

On the basis of this encouraging study, a phase II trial was performed in patients with generalised anxiety disorder (GAD) comparing XBD173 versus placebo. In this unpublished study, XBD173 showed no reduction compared with placebo in a variety of anxiety measures.

How do we explain the lack of efficacy in the GAD trial given the success with the CCK4 challenge? The answer may lie in the choice of an inappropriate disease model - the CCK4 challenge resembles panic disorder much more closely than it does GAD. But perhaps more important was a failure to appreciate differences between patients regarding the way in which the drug interacts with its target.
In early phase clinical trials there is an appropriate emphasis on pharmacokinetic differences between subjects. This is because the way in which the drug is absorbed, distributed, metabolised and excreted differs between subjects for various reasons including comorbidity (e.g. age, renal or hepatic impairment) and concomitant medication (e.g. liver enzyme inducers and inhibitors). These pharmacokinetic differences mean that to achieve a certain concentration of the drug in the plasma, some patients require substantially larger doses than others. Conversely, however, there is an assumption that the occupancy of the drug target associated with a particular concentration of the drug remains relatively constant between subjects. This is clearly an important assumption, because for most drugs the effect is mediated through occupation of a receptor, and the greater the occupation, the greater the effect. This assumption probably holds in most circumstances, because evolution would be expected to conserve the structure of important molecules (i.e. those which make attractive drug targets). Hence, if the structure of the target is conserved across subjects, then the affinity with which the drug binds to it will also be conserved, and so will the relationship between drug concentration and target occupancy.

Unfortunately, for the case of TSPO and the majority of drugs that bind it, positron emission tomography (PET) studies have 
revealed that this assumption is incorrect. As well as being an attractive drug target, TSPO is also an attractive target in PET because it is present in large numbers in microglia, the inflammatory cell whose proliferation accompanies many diseases in the brain (Venneti et al., 2006). Quantifying TSPO, and thus microglia, would be an extremely powerful research tool to understand the kinetics of neuroinflammation. However, TSPO PET studies in humans show unexpected variation in signal (Kreisl et al., 2010), which was recently demonstrated to be due to variation in the affinity with which radioligands bind the TSPO (Owen et al., 2010, 2011a). These binding affinity differences are determined by a single nucleotide polymorphism in the gene encoding the TSPO which causes a substitution of alanine to threonone at amino acid 147 (A147T) (Owen et al., 2011c). This substitution changes the configuration of the binding site at which the majority of TSPO radioligands bind (Owen et al., 2011a, 2011c). Expression of this genetic variant is co-dominant: subjects either express two copies of the wildtype allele (AA), two copies of the mutant allele (TT), or are heterozygotes and express both wildtype and mutant copies of the TSPO in 50:50 proportion (AT) (Owen et al., 2011c). In Caucasians, the mutant allele (T) is common; $9 \%$ of the population are TT and $42 \%$ are AT (Owen et al., 2011c). In the Asian population, however, the mutant allele is much less common; less than $1 \%$ are AT and TT subjects are rarer still (Kurumaji et al., 2000).

As with the majority of TSPO radioligands, XBD173 binding is also affected by the A147T substitution - the polymorphism reduces XBD173 binding affinity to TSPO by a factor of roughly 15-fold (Owen et al., 2011b) . This is highly relevant to the failed XBD173 GAD study, as the preceding XBD173 CCK4 study showed efficacy only at the highest dose of $90 \mathrm{mg}$, with no effect seen at doses of $30 \mathrm{mg}$ and below (Rupprecht et al., 2009). The 15 -fold reduction in binding affinity means that the same dose will produce huge differences in TSPO occupancy between subjects who differ with respect to the A147T substitution. For example, if we arbitrarily assume that the highest dose of XBD173 (90mg, which was sufficient to produce anxiolysis) causes TSPO occupancy of $50 \%$ in an AA subject, we can calculate that the lower dose (30mg, which did not produce an effect) would cause $25 \%$ occupancy. In this example, therefore, efficacy is reached somewhere between 25 and 50\% TSPO occupancy. However, for non-AA subjects, the reduction in binding affinity means that a $90 \mathrm{mg}$ dose would cause TSPO occupancy of $12 \%$ in an AT subject and $6 \%$ in a TT subject. Hence the dose which is efficacious in AA subjects is sub-therapeutic in the rest of the population.

The clinical trial of XBD173 in GAD was performed in a chiefly Caucasian population. Because $50 \%$ of Caucasians express at least one copy of the mutant allele, this means that half of the subjects in the XBD173 arm of the study were in fact receiving a sub-therapeutic dose of the drug. In hindsight, therefore, the lack of efficacy ultimately found is unsurprising. Repeating the study with dosing appropriate to genotype may yield more promising results. An alternative approach would be to enrol only those subjects likely to respond (i.e. AA subjects).

This recent example illustrates the importance of genotype in determining drug actions; moreover it shows how a clinical trial can be undermined by a lack of understanding of the target variability. Examples in other branches of medicine exist too, such as the presence of 2D6 poor metabolisers, who benefit less from tramadol than extensive metabolisers, as tramadol is a pro-drug that requires metabolism for full efficacy (Poulsen et al., 1996). It is likely that there are many similar examples in psychiatry treatments that have yet to be discovered, and this should be a major focus for research. Regulators should now begin to develop effective and functional rules for this new approach to stratified medicines.

\section{Acknowledgements}

The authors wish to thank Paul Jenner from Novartis for his assistance with work which contributed to this manuscript.

\section{Funding}

This work was supported by the Wellcome Trust GSK Translational Medicine Fellowship Programme (to DRO).

\section{Conflict of interest}

The authors declare that there are no conflict of interest.

\section{References}

Baldwin DS, Anderson IM, Nutt DJ, et al. (2005) Evidence-based guidelines for the pharmacological treatment of anxiety disorders: Recommendations from the British Association for Psychopharmacology. $J$ Psychopharmacol 19: 567-596.

Baulieu EE, Robel P and Schumacher M (2001) Neurosteroids: Beginning of the story. Int Rev Neurobiol 46: 1-32.

Brot MD, Akwa Y, Purdy RH, et al. (1997) The anxiolytic-like effects of the neurosteroid allopregnanolone: Interactions with GABA(A) receptors. Eur J Pharmacol 325: 1-7.

Carboni E, Wieland S, Lan NC, et al. (1996) Anxiolytic properties of endogenously occurring pregnanediols in two rodent models of anxiety. Psychopharmacology (Berl) 126: 173-178.

Gavish M, Bachman I, Shoukrun R, et al. (1999) Enigma of the peripheral benzodiazepine receptor. Pharmacol Rev 51: 629-650.

Kreisl WC, Fujita M, Fujimura Y, et al. (2010) Comparison of [(11)C](R)-PK 11195 and [(11)C]PBR28, two radioligands for translocator protein $(18 \mathrm{kDa})$ in human and monkey: Implications for positron emission tomographic imaging of this inflammation biomarker. Neuroimage 49: 2924-2932.

Kurumaji A, Nomoto H, Yoshikawa T, et al. (2000) An association study between two missense variations of the benzodiazepine receptor (peripheral) gene and schizophrenia in a Japanese sample. J Neural Transm 107: 491-500.

Nutt DJ, Ballenger JC, Sheehan D, et al. (2002) Generalized anxiety disorder: Comorbidity, comparative biology and treatment. Int J Neuropsychopharmacol 5: 315-325.

Owen DR, Howell OW, Tang SP, et al. (2010) Two binding sites for [(3)H] PBR28 in human brain: Implications for TSPO PET imaging of neuroinflammation. J Cereb Blood Flow Metab 30(9): 1608-1618.

Owen DR, Gunn RN, Rabiner EA, et al. (2011a) Mixed-affinity binding in humans with 18-kDa translocator protein ligands. J Nucl Med 52: 24-32.

Owen DR, Lewis AJ, Reynolds R, et al. (2011b) Variation in binding affinity of the novel anxiolytic XBD173 for the $18 \mathrm{kDa}$ translocator protein in human brain. Synapse 65: 257-259.

Owen DR, Yeo AJ, Gunn RN, et al. (2011c) An 18-kDa translocator protein (TSPO) polymorphism explains differences in binding affinity of the PET radioligand PBR28. J Cereb Blood Flow Metab 32(1): 1-5.

Papadopoulos V (1993) Peripheral-type benzodiazepine/diazepam binding inhibitor receptor: Biological role in steroidogenic cell function. Endocr Rev 14: 222-240.

Papadopoulos V, Amri H, Li H, et al. (1997) Targeted disruption of the peripheral-type benzodiazepine receptor gene inhibits steroidogenesis in the R2C Leydig tumor cell line. J Biol Chem 272: 32129-32135. 
Poulsen L, Arendt-Nielsen L, Brosen K, et al. (1996) The hypoalgesic effect of tramadol in relation to CYP2D6. Clin Pharmacol Ther 60: 636-644.

Rone MB, Fan J and Papadopoulos V (2009) Cholesterol transport in steroid biosynthesis: Role of protein-protein interactions and implications in disease states. Biochim Biophys Acta 1791: 646-658.

Rupprecht R and Holsboer F (1999) Neuroactive steroids: Mechanisms of action and neuropsychopharmacological perspectives. Trends Neurosci 22: 410-416.

Rupprecht R, Rammes G, Eser D, et al. (2009) Translocator protein (18 $\mathrm{kD})$ as target for anxiolytics without benzodiazepine-like side effects. Science 325: 490-493.

Rupprecht R, Papadopoulos V, Rammes G, et al. (2010) Translocator protein $(18 \mathrm{kDa})(\mathrm{TSPO})$ as a therapeutic target for neurological and psychiatric disorders. Nat Rev Drug Discov 9: 971-988.
Strohle A, Romeo E, di Michele F, et al. (2003) Induced panic attacks shift gamma-aminobutyric acid type A receptor modulatory neuroactive steroid composition in patients with panic disorder: Preliminary results. Arch Gen Psychiatry 60: 161-168.

Venneti S, Lopresti BJ and Wiley CA (2006) The peripheral benzodiazepine receptor (translocator protein $18 \mathrm{kDa}$ ) in microglia: From pathology to imaging. Prog Neurobiol 80: 308-322.

Verleye M, Akwa Y, Liere P, et al. (2005) The anxiolytic etifoxine activates the peripheral benzodiazepine receptor and increases the neurosteroid levels in rat brain. Pharmacol Biochem Behav 82: 712-720.

Woods MJ, Zisterer DM and Williams DC (1996) Two cellular and subcellular locations for the peripheral-type benzodiazepine receptor in rat liver. Biochem Pharmacol 51: 1283-1292. 